\title{
Oosterhoff I and II RR Lyrae Variable Masses
}

\author{
Arthur N. Cox
}

Los Alamos Astrophysics

\begin{abstract}
The anomalously low theoretical masses for double-mode $\mathrm{R} R$ Lyrae variable stars obtained from accurately observed period ratios recently have been increased by using stellar models constructed with the new Livermore OPAL opacities. These new models appear to the lowest order radial modes to be less concentrated in density, and they thus produce longer periods. Since the fundamental mode is increased in period more than the first overtone, this period ratio from the model is decreased. Cox (1991) showed that now these pulsation-based masses agree with evolution masses, and all RR Lyrae variable stars show little mass loss from the red giant tip in earlier evolution. This investigation has been validated by two additional papers that use more directly the OPAL opacities rather than simple adjustments to the Stellingwerf (1975ab) fit. These new papers (Kovacs, Buchler, and Marom, 1991, and Kovacs, Buchler, Marom, Iglesias, and Rogers, 1992) also display composition effects for Population II mixtures.

My new study uses directly the latest OPAL intermediate coupling opacities for the Oosterhoff $I$ and II ( $\mathrm{Z}=0.0003$ and 0.0001 , respectively) compositions in frozen-in convection (mixing length/pressure scale height ratio=1.25) models to see if there is any difference between the masses of variable stars in these two globular cluster classes. Derived masses are just over 0.65 and $0.75 M_{\odot}$, respectively. The Oosterhoff I mass could be as large as the Oosterhoff II mass only if the iron abundance in the $\mathrm{Z}$ mixture is increased by more than $30 \%$ over that for the solar mixture.

The Sandage effect of higher periods for variables in Oosterhoff II clusters at lower $\mathrm{Z}$ than for the Oosterhoff I clusters might be explained with my new masses by having the Oosterhoff II variables born much to the blue of the instability strip and evolving at increasing luminosity to the red. For Oosterhoff I variables, they can be born at their lower mass and higher $Z$ inside the instability strip at lower luminosity, evolving both to the red and blue with little evolutionary luminosity increase before they become red nonvariables.
\end{abstract}

\section{References:}

Cox, A. N., 1991, Ap. J. Lett., 381, L71.

Kovacs, G., Buchler, J. R., and Marom, A., 1991, Astron. Astrophys., 252, L27.

Kovacs, G., Buchler, J. R., Marom, A., Iglesias, C. A. and Rogers, F. J., 1992, Astron. Astrophys., in press.

Stellingwerf, R. F., 1975, Ap. J. 195, 441.

Stellingwerf, R. F., 1975, Ap. J. 199, 705. 